\title{
Hippocampal Neurons Encode Different Episodes in an Overlapping Sequence of Odors Task
}

\author{
Matthew R. Ginther, ${ }^{1}$ Devin F. Walsh, ${ }^{1}$ and Seth J. Ramus ${ }^{1,2}$ \\ ${ }^{1}$ Program in Neuroscience, and ${ }^{2}$ Department of Psychology, Bowdoin College, Brunswick, Maine 04011
}

Recent theoretical models of hippocampal function suggest that the hippocampus plays a critical role in the memory for the overlapping sequences of events that comprise episodic memory. Consistent with this idea, the firing of hippocampal "place cells" have been shown to represent not only location, but also the context or episode in which the location occurs. Thus, hippocampal neurons fire differently in the same location depending on the particular journey or sequence of places in which the subject is traveling. Further, recent work in rats has shown that hippocampal lesions impair memory for sequences of odors and the ability to disambiguate overlapping sequences of odors. We therefore recorded the activity of hippocampal complex-spike cells during a disambiguation of odor sequences task in which the two sequences shared three common odors. Consistent with data from spatial memory tasks, we found that 26 of 44 complex-spike cells fired differentially in the periods before, or during the presentation of the ambiguous odors depending on the sequence in which the odors were presented. This finding further supports the idea that the hippocampus is critical for episodic memory, and extends the physiological evidence to suggest that the hippocampal neurons play a broader role representing sequences of both spatial and nonspatial information.

\section{Introduction}

In humans, the hippocampus is critically involved in the memory for distinct personal experiences (Scoville and Milner, 1957; Vargha-Khadem et al., 1997) or episodic memory (Tulving, 1983). Some elements of episodic memory can be tested in animals. Specifically, animals do have the ability to recall information within an event's spatial and temporal context, or "episodic-like" memory (Clayton and Dickinson, 1998; Ergorul and Eichenbaum, 2004; Babb and Crystal, 2006).

Hippocampal neurons encode the experience of odors independently, and within the context of their spatial locations (Wood et al., 1999; Komorowski et al., 2009). Further, even hippocampal "place cells" encode spatial locations within the context of different memory episodes (Frank et al., 2000; Wood et al., 2000; Ferbinteanu and Shapiro, 2003; Lee et al., 2006; Smith and Mizumori, 2006). Additionally, behavioral studies in rats have demonstrated that the hippocampus plays a critical role in the memory for nonspatial sequences of odors (Fortin et al., 2002; Kesner et al., 2002) and, importantly, that the hippocampus is necessary for the successful disambiguation of overlapping sequences of odors (Agster et al., 2002). Most recently, Brown et al. (2010) have shown greater activation in the hippocampus as human subjects successfully navigate overlapping mazes. These findings have lent considerable evidence to support a theory of

Received July 1, 2010; revised Nov. 22, 2010; accepted Dec. 17, 2010.

This work was supported by National Institute of Mental Health Grant 1R15MH075755 and the Howard Hughes Medical Institute (M.R.G., D.F.W.). We thank the Eichenbaum laboratory at Boston University for their time and assistance, particularly Norbert Fortin, Daniel Salz, Rob Komorowski, and Ben Kraus. We would also like to thank Joe Manns at Emory University for his significant assistance and guidance.

Correspondence should be addressed to Seth J. Ramus, Bowdoin College, Department of Psychology, 6900 College Station, Brunswick, ME 04011. E-mail: sramus@bowdoin.edu.

DOI:10.1523/JNEUROSCI.3413-10.2011

Copyright $\odot 2011$ the authors $\quad 0270-6474 / 11 / 312706-06 \$ 15.00 / 0$ hippocampal function in which the hippocampus processes both spatial and nonspatial elements based on their sequential order to create and maintain the temporal and spatial relationship between distinct, yet overlapping, episodes (Eichenbaum et al., 1999; Hasselmo and Eichenbaum, 2005; Zilli and Hasselmo, 2008).

This current study extends the physiological evidence for this model from spatial to nonspatial domains by describing the firing of hippocampal complex-spike cells during the disambiguation of two nonspatial sequences of odors. This task is known to be hippocampal dependent (Agster et al., 2002) and is formally similar to the spatial alternation on the T-maze task described by Wood et al. (2000) (Fig. 1A). Two overlapping sequences of seven odors were randomly presented in series. The two sequences shared the three middle odors (sequence 1: MNABCOP; sequence 2: WXABCYZ). However, for one of the odor sequences (sequence 2), the common odors ( $\underline{\mathrm{ABC}}$ ) were not rewarded. Thus, the reward was predictable only through the ability to disambiguate between the two sequences. As in the T-maze task, the majority of hippocampal neurons were selective for the context in which the ambiguous stimuli appeared. These "splitter" or "episodic memory" cells are consistent with a more general model of hippocampal function in which hippocampal neurons represent both spatial and nonspatial elements within the contexts in which they occur.

\section{Materials and Methods}

Subjects and surgery. Subjects were five male Long-Evans rats, weighing $350-400 \mathrm{~g}$ at the beginning of testing. Rats were housed individually on a 14/10 $\mathrm{h}$ light/dark cycle, and given ad libitum access to chow and water until training, at which point access to water was restricted to $30 \mathrm{~min}$ per day in addition to the water received as reward $(\sim 8 \mathrm{ml})$. Body weight was monitored to ensure a minimum of $90 \%$ normal body weight. All pro- 

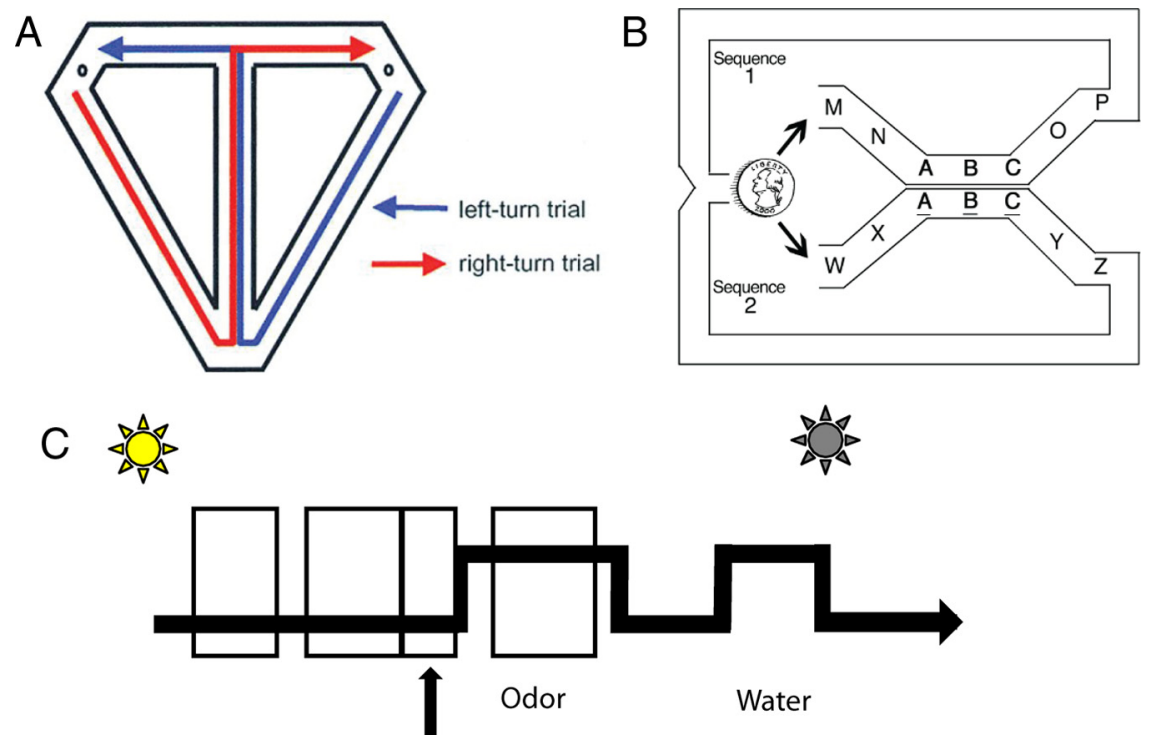

Figure 1. Two disambiguation of overlapping sequences tasks. $A$, The T-maze alternation task described by Wood et al. (2000, their Fig. 1A). In this task, rats ran a figure eight pattern traversing the central arm on both left and right turn trials. Hippocampal place cells with firing fields on the central arm fired differently depending on whether the rat was going to make a left or a right turn. $\boldsymbol{B}$, The overlapping odor sequence task used in the current study is formally similar to that in $\boldsymbol{A}$, using sequences of odors instead of sequences of places. Sequences were presented continuously and randomly (coin). 0 dors are represented by letters, and the two sequences shared the common odors $\mathrm{ABC}$. All odors were rewarded except the odors $\mathrm{ABC}$ in sequence 2 (underlined). Thus, whether a shared odor was rewarded was determined by the sequence in which the odor occurred. $C$, Timeline of a single trial. On a given trial, the houselight turns on indicating that the rat may nosepoke in the odor port (arrow). After a variable delay, the odor is delivered. If the rat maintains the nosepoke for at least $1250 \mathrm{~ms}$, a nosepoke in the water port would result in the delivery of water, extinction of the houselight, and initiation of a 3 s intertrial interval. Analysis epochs are indicated by boxes that include (left to right) the preodor period (1500-1000 ms before the odor poke), approach period ( $800-200 \mathrm{~ms}$ before the odor poke), odor-poke period ( $200 \mathrm{~ms}$ before to $200 \mathrm{~ms}$ after the odor poke), and odor-sampling period ( $200-1000 \mathrm{~ms}$ after the odor onset).

cedures were approved by the Bowdoin College Research Oversight Committee.

Before training, rats were implanted with chronic, drivable, extracellular electrodes. Immediately before surgery, the animal was given an intramuscular injection of an antibiotic, enrofloxacin $(0.02 \mathrm{ml} / \mathrm{kg})$. Anesthesia was initiated at $3-5 \%$ isoflurane and maintained at $1-2 \%$ for the duration of the surgery. Anesthesia was supplemented with subcutaneous administrations of butorphanol at a dosage of $0.01 \mathrm{ml} / 100 \mathrm{~g}$ per $4 \mathrm{~h}$, and atropine sulfate at a dosage of $0.1 \mathrm{ml}$ per $4 \mathrm{~h}$ intramuscularly to control respiratory secretions. Once at a surgical plane of anesthesia, the skull was exposed and small holes drilled above the dorsal hippocampus, and the dura was removed, exposing the pial surface of the brain. Electrodes were subsequently lowered at a rate of $0.1 \mathrm{~mm}$ every $30 \mathrm{~s}$ to a target in the dorsal region of hippocampal CA1 (anterior-posterior: -3.2; medial-lateral: 2.0). Electrode drivers were attached to the skull using dental cement anchored to five to seven screws inserted into the skull. The rats were given a minimum of 1 week for postsurgery recovery, during which acetaminophen and enrofloxacin were administered in suspension with drinking water.

Electrophysiological recording. Hippocampal recordings were accomplished using a custom-built "hyperdrive" with eight independently drivable tetrodes (Manns et al., 2007). Each tetrode was composed of four $12.5 \mu \mathrm{m}$ Formvar-coated nichrome wires (California Fine Wire). The structure of the tetrode was maintained by a brief application of heat to meld the electrode insulation together. Each electrode's impedance was reduced to $200-250 \mathrm{k} \Omega$ by electroplating each electrode tip with gold by passing $2 \mathrm{~mA} \mathrm{DC}$ across the electrode while the electrode tip was submerged in a gold suspension (SIFCO). Final impedance was measured at $1 \mathrm{kHz}$. Tetrodes were sheathed in polyimide tubing and driven into tissue using a custom mold (Boston University Machine Shop). Tetrode wires interfaced onto a 64-channel EIB chip (EIB-64, Neuralynx) with two dedicated ground wires fastened to head screws inserted above the cerebellum.
Hippocampal cells were identified, and the correct placement of the electrodes was confirmed though the hallmark electrophysiological characteristics of hippocampal unit activity. These hallmarks have been well established and consisted of complex spikes and multiple-cell "bursts" accompanied by $\sim 200 \mathrm{~Hz}$ ripples in the field potential (Buzsáki, 2002). Trial activity was recorded only when clear hippocampal units could be isolated that day. Any cell that appeared across multiple trials was analyzed only once. Following each recording session, tetrodes were advanced $\sim 50 \mu \mathrm{m}$ and allowed to rest overnight. At the conclusion of the experiment, the placement of the electrodes was confirmed histologically. In all cases, the electrode bundles traversed the CA1 subfield of the dorsal hippocampus.

Neural activity was first processed through unity gain amplifiers in the headstage of the recording cable (Plexon o50 Headstage, Plexon). Signals were then differentially amplified and bandpass filtered $(10,000 \times$, filtered for $600-6000 \mathrm{~Hz}$ ) using a 16-channel Multichannel Acquisition Processor (Plexon). Unit activity was stored using Plexon Sort Client software, and individual spikes were isolated off-line using multiple spike parameters, including Plexon proprietary waveform characterization functions.

Behavioral testing. Training and testing both occurred in a custom $30 \times 30 \mathrm{~cm}$ aluminum operant chamber. The chamber contained an odor port and an adjacent water port. Proximity to these ports was monitored with photodetectors. The operant chamber was illuminated by a houselight that indicated trial onset and extinguished at the end of each trial. Odors were administered, under computer control, by the opening and closing of solenoids, leading to a continuously running plain air source attached to the port. The odors were evacuated from the port by means of vacuum pressure. Full details of the testing apparatus can be found in Ramus and Eichenbaum (2000). Odors were 11 commercially available imitation and natural food extracts diluted to a concentration just noticeable to the experimenter $(\sim 1: 100)$. The odors were anise, coconut, cherry, lemon, orange, almond, vanilla, and banana, strawberry, maple, and rum.

Initially, rats were trained to insert their snouts into the odor port to receive a water reward. During this phase of training, no odors were presented, and a poke in the odor port resulted in delivery of clean air. Rats were trained successfully poke for the $1250 \mathrm{~ms}$ (criteria of 90 of 100 trials) before beginning the overlapping sequence task.

In the overlapping sequence task, rats were presented with two separate sequences of seven odors each (sequence 1: MNABCOP; sequence 2: WXABCYZ) (Fig. 1B,C). The two sequences shared the three middle odors (ABC), which were presented identically to ensure that the odors could not be characterized by any means other than their sequential context. On a given trial, the houselight would turn on indicating that the rat could poke in the odor port. A poke would initiate a variable 150-350 ms delay before the delivery of the odor. After holding in the port for $1250 \mathrm{~ms}$, a poke in the water port would result in the delivery of water, extinction of the houselight, and initiation of a $3 \mathrm{~s}$ intertrial interval. Withdrawal from the odor port before the $1250 \mathrm{~ms}$ would result in the immediate extinction of the houselight and initiation of a $5 \mathrm{~s}$ intertrial interval. Once the rat completed all seven trials of a given sequence, the next sequence would be determined randomly, and the next seven trials would begin immediately without a delay or cue indicating the start of a new sequence. Recording sessions consisted of $40-70$ sequences $(\sim 300-$ 500 trials). Rats were rewarded with $\sim 0.03 \mathrm{ml}$ of water following a successful poke into the odor port after each odor in both sequences except for odors $\underline{\mathrm{ABC}}$ when they appeared as part of sequence 2 . 


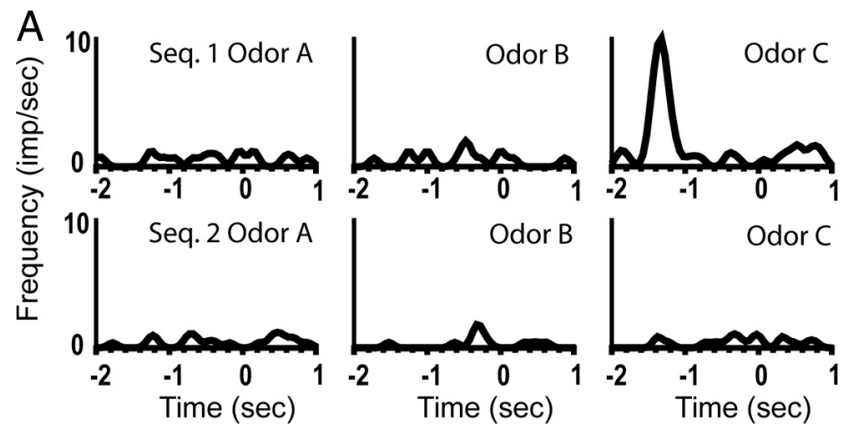

B

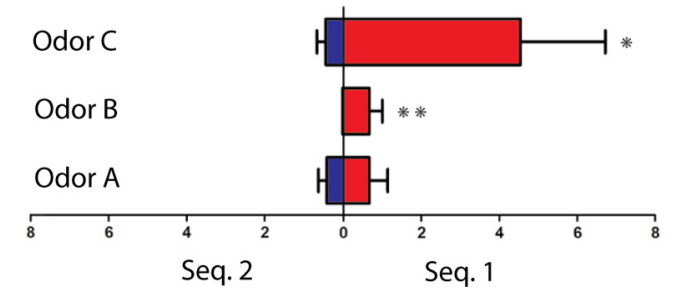

Figure 2. An example of a splitter cell that fired differently before the presentation of the ambiguous odors during the preodor period. $\boldsymbol{A}$, Line histograms illustrating the activity of cell M11-11/10.9a from 2 s before odor poke to $1 \mathrm{~s}$ after odor poke. Top row: average activity of the neuron to the ambiguous odors in the rewarded sequence (sequence $1 ;$ odors A-C, left to right). Bottom row: average activity to the same odors in the unrewarded sequence. This neuron showed a significant main effect of sequence and a significant sequence $\times$ odor interaction. This neuron also showed a significant sequence $X$ odor interaction during the odor-sampling period. $\boldsymbol{B}$, Average firing rate \pm SEM during the preodor period for the cell presented in $\boldsymbol{A}$. Left bars indicate the mean firing rate in sequence 2 , and the right bars in sequence 1. Bottom: odor A; middle: odor B; top: odor C. Post hoc analyses: ${ }^{*} p<0.05,{ }^{* *} p<0.01,{ }^{* * *} p<0.001$. Seq., Sequence.

\section{Results}

Since this task was intended to be formally similar to the overlapping sequence of places task, the analysis of neuronal activity was adapted from Wood et al. (2000) to make the results comparable. Analysis focused on neurons that showed clear activity corresponding with the presentation of the ambiguous odors (ABC) common to the two sequences. A total of 57 neurons displaying characteristics of hippocampal complex-spike cell activity were isolated from five rats as they were presented with the two overlapping sequences of seven odors (Fig. 1B,C). A typical session yielded $4.4 \pm 1.0$ cells during the presentation of an average of $45 \pm 5.4$ complete sequences. In the first stage of analysis, mean firing rates were calculated for each neuron during three time epochs corresponding with the nosepoke into the odor port or the odor onset (Fig. 1C). These epochs included the period before the poke into the odor port, approach (800-200 ms before the odor poke), the odor poke (200 ms before to $200 \mathrm{~ms}$ after the odor poke), and the odor-sampling period (200$1000 \mathrm{~ms}$ after the odor onset). Of the 57 complex-spike cells, 43 showed robust activity (defined as $3 \times$ the mean firing rate) corresponding with the onset of the ambiguous odors during approach $(n=18)$, odor poke $(n=20)$, odor sampling $(n=$ 14 ), or a combination of epochs. During preliminary examination, we noted a class of cells that showed transient increases in firing rate at times $>1000 \mathrm{~ms}$ before the odor poke and did not correlate with any other behavioral event (e.g., onset of the houselight) (Fig. 2). We therefore added a fourth epoch to the analysis, the preodor period (1500-1000 ms before the odor poke). A total of 13 cells showed robust activity during this epoch, bringing the total number of cells showing robust changes during one of the four epochs to 44 .
In a second stage of analysis, the mean firing rates of the 44 neurons were compared with determine whether there was differential firing dependent on the sequence in which the odors were presented. For each cell, we used a $2 \times 3$ (sequence $\times$ odor) ANOVA to compare the firing rates of each neuron during any epoch in which the cell showed robust firing. Of the 44 neurons, 26 (59\%) fired differentially between two odor sequences $(p<$ 0.05 ) or between odor sequence as a function of odor (sequence $\times$ odor interaction, $p<0.05$ ). Four of the 26 neurons showed differential firing during two periods, usually adjacent epochs, although one neuron (M11-11/10.9a) showed differential firing during the preodor period and the odor-sampling period. This neuron is illustrated in Figure 2 and described in detail below in Preodor period. Thus, these 26 complex-spike cells showed differential firing dependent on the sequence in which the odors were presented.

\section{Preodor period}

Of the 13 neurons that showed robust firing during the preodor period, $8(62 \%)$ showed a significant main effect of sequence or a significant sequence $\times$ odor interaction. As noted above, firing during this epoch was typically transient and was not related to any other behavioral event (e.g., the onset of the houselight). For example, the firing of neuron M11-11/10.9a is illustrated in Figure 2. This neuron showed a significant main effect of sequence $\left(F_{(1,66)}=6.31, p<0.05\right)$, indicating that the neuron fired differently during the period between 1500 and $1000 \mathrm{~ms}$ before the odor poke, depending on which sequence was presented. Additionally, the neuron showed a significant sequence $X$ odor interaction $\left(F_{(2,66)}=3.12, p=0.05\right)$, indicating that the firing was modulated by the identity of the odors. This neuron also showed a significant sequence $X$ odor interaction during the odorsampling period $\left(F_{(2,68)}=4.27, p<0.05\right)$, although the magnitude of the change in firing was greater during the preodor period ( $14 \times$ baseline) than during the odor-sampling period $(3 \times$ baseline). In this example, the neuron showed the maximal change in firing before or during the presentation of odor $\mathrm{C}$ of the rewarded sequence (sequence 1) during both epochs.

\section{Approach period}

Of the 18 neurons that showed robust firing during the approach period, $10(56 \%)$ showed a significant main effect of sequence or a significant sequence $\times$ odor interaction. For example, the firing of neuron M5-04/15.13a is illustrated in Figure 3. This neuron showed a significant main effect of sequence $\left(F_{(1,177)}=12.12, p<\right.$ $0.001)$ and a significant sequence $\times$ odor interaction $\left(F_{(2,177)}=\right.$ $7.34, p<0.001)$, indicating that the neuron fired differently during the epoch 800-200 ms before the odor poke, depending on the identity of the odors and in which sequence the odors were presented.

\section{Odor-poke period}

Of the 20 neurons that showed robust firing during the odorpoke period, $9(45 \%)$ showed a significant main effect of sequence or a significant sequence $\times$ odor interaction. For example, the firing of neuron M10-10/30.13d is illustrated in Figure 4 . This neuron demonstrated a significant main effect of sequence $\left(F_{(1,41)}=7.66, p<0.01\right)$, firing more during the epoch $200 \mathrm{~ms}$ before to $200 \mathrm{~ms}$ after the odor poke in the rewarded than the unrewarded sequence.

\section{Odor-sampling period}

Of the 14 neurons that showed robust firing during the odorsampling period, only $3(21 \%)$ showed a significant main 


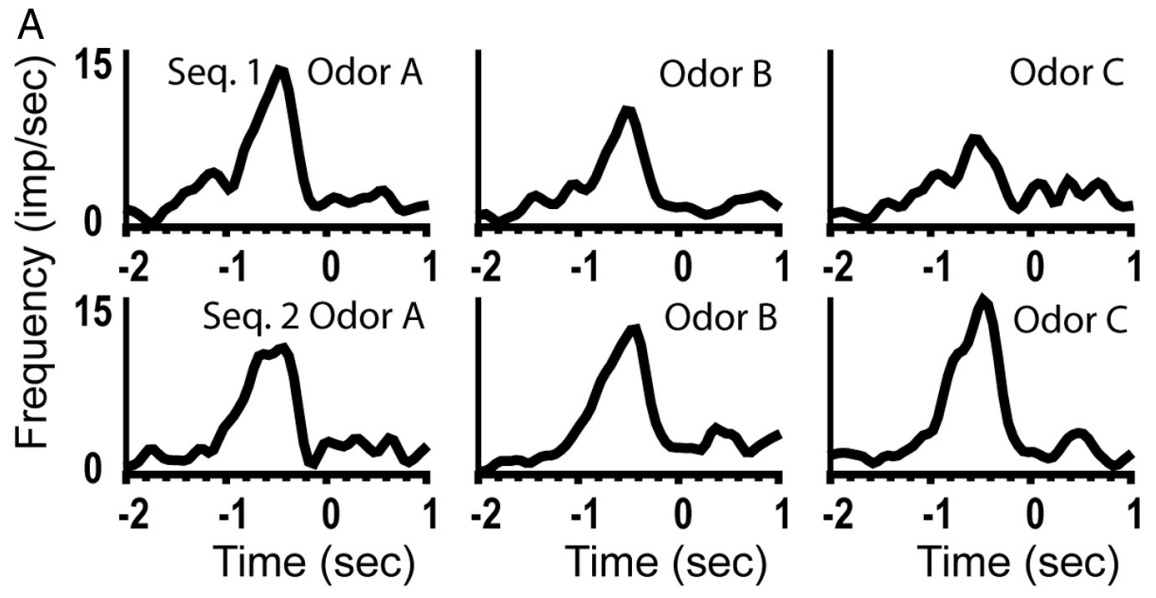

B

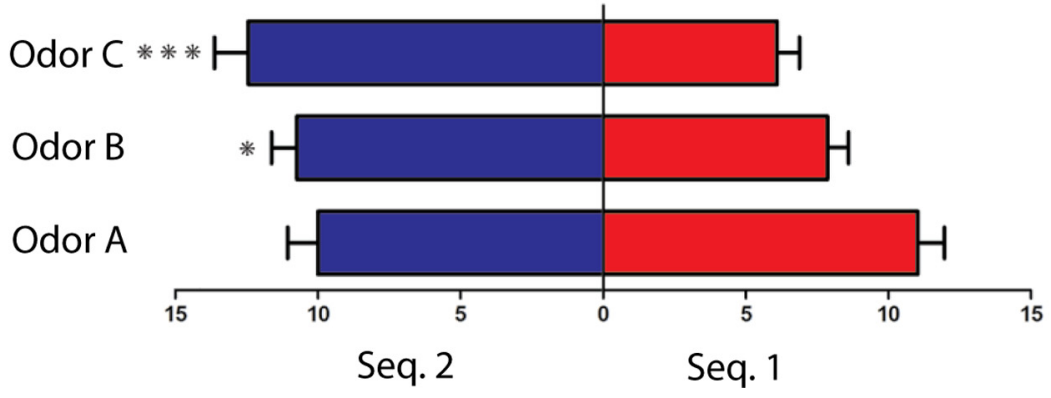

Figure 3. An example of a splitter cell that fired differently before the presentation of the ambiguous odors during the approach period. $A$, Line histograms illustrating the activity of cell M5- 04/15.13a from $2 \mathrm{~s}$ before odor poke to $1 \mathrm{~s}$ after odor poke. Top row: average activity of the neuron to the ambiguous odors in the rewarded sequence (sequence $1 ;$ odors $A-C$, left to right). Bottom row: average activity to the same odors in the unrewarded sequence. This neuron showed a significant main effect of sequence and a significant sequence $X$ odor interaction. $\boldsymbol{B}$, Summary of the average firing rate during the preodor period for the cell presented in $A$. Left bars indicate the mean firing rate in sequence 2 , and the right bars in sequence 1 . Bottom: odor A; middle: odor $B$; top: odor C. Post hoc analyses: ${ }^{*} p<0.05,{ }^{* *} p<0.01,{ }^{* * *} p<0.001$. Seq., Sequence.

effect of sequence or a significant sequence $\times$ odor interaction. In general, the magnitude of response of these neurons was weaker than for the other three epochs (e.g., neuron M1111/10.9a illustrated in Fig. 2 and described in the Preodor period section).

\section{Discussion}

Hippocampal complex-spike cells were recorded as rats were presented with two overlapping sequences of odors. The two sequences shared the three middle odors (sequence 1: MNABCOP; sequence 2: WXABCYZ; see Materials and Methods). However, for one of the odor sequences (sequence 2), the common odors $(\underline{\mathrm{ABC}})$ were not rewarded. Thus, the reward was predictable only through the ability to disambiguate between the two sequences. Of the 57 complex-spike cells recorded in this task, 44 (77\%) showed robust activity during portions of the sequence associated with the three ambiguous odors. Importantly, 26 of the 44 complex-spike cells (59\%) fired differentially in the periods before or during the presentation of the ambiguous odors depending on the sequence in which the odors were presented.

That the hippocampal neurons demonstrated significant differences in firing during analysis epochs associated with the same odor as it appeared in the two sequences likely reflects the mnemonic demands of the task. In other words, to know whether an ambiguous odor will be rewarded, the rat needed to remember the sequence in which the odor appeared. This sequencedependent representation of odors is consistent with findings that hippocampal lesions impair a rat's ability to learn sequences of odors (Fortin et al., 2002; Kesner et al., 2002), to disambiguate overlapping sequences of odors (Agster et al., 2002), and that the firing of complex-spike cells represents the odors within the context in which they were presented (Wood et al., 1999; Komorowski et al., 2009).

Similar to our findings, hippocampal place cells have also been shown to encode spatial locations within the context of different memory episodes (Frank et al., 2000; Wood et al., 2000; Ferbinteanu and Shapiro, 2003; Lee et al., 2006). For example, Wood et al. (2000) trained rats on a spatial alternation in a T-maze task, a task formally similar to the one presented in this article (Fig. $1 A$ ). Thus, the ambiguous odors in the overlapping sequence are analogous to the ambiguous places on the stem (an overlapping sequence of places) of the T-maze. In the Wood et al. (2000) study, 22 of the 33 cells (66\%) with place fields on the stem of the maze fired differentially based on whether the rat was going to make a right or a left turn. This percentage of cells is comparable to the proportion of splitter cells or episodic memory cells found in our current study (59\%).

Unlike Wood et al. (2000), the splitter cells in the present study were not evenly distributed across the three ambiguous odors. Of the 26 cells, 5 fired maximally to odor A, 6 to odor B, and 15 to odor C. Thus, the majority of cells demonstrated a preference for epochs before the third and final ambiguous odor $\left(\chi^{2}(2)=7.00, p<\right.$ $0.05)$. Further, the rewarded sequence was also disproportionately represented. Twenty-one of the 26 cells fired maximally to the rewarded odors, while 5 fired to the unrewarded odors $\left(\chi^{2}(1)=9.845, p<0.01\right)$. An important difference between the current study and previous studies examining ambiguous journeys is that the rats were rewarded after the presentation of the ambiguous odors in the context of one sequence but not in the context of the other. So, one possibility is that the differential firing could represent the delivery of the water reward. However, this explanation is unlikely because of the way that the task was implemented. First, the intertrial interval was a minimum of $3 \mathrm{~s}$, and the rats quickly visited the water port after leaving the odor port. Second, the task required that the rat was out of the water port before the next trial initiated. And finally, it would be hard to explain why the cells disproportionately responded to the third rewarded odor, and not to the other two rewarded odors (e.g., the cell represented in Fig. 2), if the cells were simply representing the reward. Additionally, 11 of the 26 splitter cells also represented the identity of odor, either as a main effect of odor $(n=5)$, a significant odor $\times$ sequence interaction $(n=5$; the cell represented in Fig. 3), or both $(n=1)$.

A second possibility is that the differential firing may represent the motivational state of the animal. In fact, Kennedy and Shapiro (2009) have recently shown that the firing of hippocampal neurons during a common journey in a nonspatial contextual memory task is modulated by whether the rat was hungry or thirsty. They found cells that fired in the same location but at 
different rates, depending on the deprivation condition, while other neurons fired in different locations or different spatial patterns. Further, findings by Smith and Mizumori (2006) that showed that hippocampal place cells changed the location of their firing fields on a plus maze depending on where on the maze (context) the reward was presented, as well as more complex conjunctions including start position and trajectory. Therefore, a more likely explanation is that the reward or motivational state served as a context that signaled the identity of the sequence, and the cells were representing the odor identity within this context.

In this study, the majority of splitter cells was found in the epochs before the presentation of the ambiguous odors. Eight neurons demonstrated differential firing during the preodor period, 10 during the approach period, and 9 during the odor poke. We had expected the cellular response during the odor-sampling epoch to most closely reflect the cellular response seen in Wood et al. (2000) as the rat traversed the place fields of the common stem. We based this prediction on the presumption that the odor-sampling epoch most closely mimicked the spatial sampling that occurs as the rat traverses the place fields of the central arm. Surprisingly, of 26 neurons demonstrating sequence differentiation, only 3 were from the sampling period.

However, previous evidence has suggested that hippocampal neurons support the representation of learned sequences of odors in the period before the odor onset. For example, we have shown that neurons in the orbitofrontal cortex (OFC) show odorselective firing before odor onset when rats learned a single, repeated sequence of odors. Further, this odor-selective anticipatory firing depended on the integrity of the hippocampus (Ramus et al., 2007). Thus, although these odor sequence tasks are not identical, it seems likely that the OFC and hippocampus both represent learned sequences of odors during the period before odor onset. More recently, a human functional magnetic resonance imaging study by Brown et al. (2010) has also implicated both the OFC and the hippocampus in the disambiguation of overlapping trajectories (sequences of places). Principally, their results demonstrated that the hippocampus showed greater activation on trials in which the subjects successfully navigated overlapping mazes compared with non-overlapping mazes. Further, the OFC was bilaterally activated when the subjects were traversing the critical overlapping halls. We suggest that the OFC may therefore integrate the sensory and contextual information necessary for the subject to make a correct behavioral response. Together, these findings suggest that the odor-specific anticipatory firing in the OFC observed by Ramus et al. (2007) might reflect the sequential information represented in the firing of the hippocampal complex-spike cells.

Thus, one final and intriguing possibility is that this anticipatory firing might represent temporal coding in the hippocampus.
For example, Pastalkova et al. (2008) have shown that hippocampal neurons fire transiently at particular times in a running wheel during the delay period of a T-maze alternation task. Further, the activity of these episode cells depended on the context of the task and predicted the future choice of the rat in the maze. Although our study was not designed to directly test this possibility, we did observe transient firing before the presentation of individual odors (e.g., the cell represented in Fig. 2), and the majority of cells with differential activity showed maximal changes in firing rate distributed across the three epochs before the presentation of the ambiguous odors. It is therefore possible that the firing of these hippocampal neurons represent "episode fields" that reflect a particular time in the sequence along with the particular behavior or expectation that occurs at that time.

In summary, our findings contribute to a recent trend of evidence supporting a model in which the hippocampus is critical for the memory of overlapping sequences of events that comprise episodic memory (Eichenbaum et al., 1999; Hasselmo and Eichenbaum, 2005; Zilli and Hasselmo, 2008). As was noted by the authors in Wood et al. (2000), the repeating stimuli presented in this task do not constitute the unique personal experiences that define episodic memory in humans (Tulving, 1983). However, our results do show that hippocampal neurons encode for the presence and expectation of nonspatial stimuli based on the motivational or temporal context of the experience. This further extends the physiological evidence for this model from sequences of overlapping places to sequences of overlapping odors, suggesting that the hippocampus plays a broader role in the representation of sequences of both spatial and nonspatial information. 


\section{References}

Agster KL, Fortin NJ, Eichenbaum H (2002) The hippocampus and disambiguation of overlapping sequences. J Neurosci 22:5760-5768.

Babb SJ, Crystal JD (2006) Episodic-like memory in the rat. Curr Biol 16:1317-1321.

Brown TI, Ross RS, Keller JB, Hasselmo ME, Stern CE (2010) Which way was I going? Contextual retrieval supports the disambiguation of well learned overlapping navigational routes. J Neurosci 30:7414-7422.

Buzsáki G (2002) Theta oscillations in the hippocampus. Neuron $33: 325-340$

Clayton NS, Dickinson A (1998) Episodic-like memory during cache recovery by scrub jays. Nature 395:272-274.

Eichenbaum H, Dudchenko P, Wood E, Shapiro M, Tanila H (1999) The hippocampus, memory, and place cells: is it spatial memory or memory space? Neuron 23:209-226.

Ergorul C, Eichenbaum H (2004) The hippocampus and memory for "what," "where," and "when." Learn Mem 11:397-405.

Ferbinteanu J, Shapiro ML (2003) Prospective and retrospective memory coding in the hippocampus. Neuron 40:1227-1239.

Fortin NJ, Agster KL, Eichenbaum HB (2002) Critical role of the hippocampus in memory for sequences of events. Nat Neurosci 5:458-462.

Frank LM, Brown EN, Wilson M (2000) Trajectory encoding in the hippocampus and entorhinal cortex. Neuron 27:169-178.

Hasselmo ME, Eichenbaum H (2005) Hippocampal mechanisms for the context-dependent retrieval of episodes. Neural Netw 18:1172-1190.

Kennedy PJ, Shapiro ML (2009) Motivational states activate distinct hippocampal representations to guide goal-directed behaviors. Proc Natl Acad Sci U S A 106:10805-10810.

Kesner RP, Gilbert PE, Barua LA (2002) The Role of the hippocampus in memory for the temporal order of a sequence of odors. Behav Neurosci 116:286-290.

Komorowski RW, Manns JR, Eichenbaum H (2009) Robust conjunctive item-place coding by hippocampal neurons parallels learning what happens where. J Neurosci 29:9918-9929.

Lee I, Griffin AL, Zilli EA, Eichenbaum H, Hasselmo ME (2006) Gradual translocation of spatial correlates of neuronal firing in the hippocampus toward prospective reward locations. Neuron 51:639-650.

Manns JR, Zilli EA, Ong KC, Hasselmo ME, Eichenbaum H (2007) Hippocampal CA1 spiking during encoding and retrieval: relation to theta phase. Neurobiol Learn Mem 87:9-20.

Pastalkova E, Itskov V, Amarasingham A, Buzsáki G (2008) Internally generated cell assembly sequences in the rat hippocampus. Science 321:1322-1327.

Ramus SJ, Eichenbaum H (2000) Neural correlates of olfactory recognition memory in the rat orbitofrontal cortex. J Neurosci 20:8199-8208.

Ramus SJ, Davis JB, Donahue RJ, Discenza CB, Waite AA (2007) Interactions between the orbitofrontal cortex and the hippocampal memory system during the storage of long-term memory. Ann N Y Acad Sci 1121:216-231.

Scoville WB, Milner B (1957) Loss of recent memory after bilateral hippocampal lesions. J Neurol Neurosurg Psychiatry 20:11-21.

Smith DM, Mizumori SJ (2006) Learning-related development of contextspecific neuronal responses to places and events: the hippocampal role in context processing. J Neurosci 26:3154-3163.

Tulving E (1983) What is episodic memory? Curr Direct Psychol Sci 2:67.

Vargha-Khadem F, Gadian DG, Watkins KE, Connelly A, Van Paesschen W, Mishkin M (1997) Differential effects of early hippocampal pathology on episodic and semantic memory. Science 277:376-380.

Wood ER, Dudchenko PA, Eichenbaum H (1999) The global record of memory in hippocampal neuronal activity. Nature 397:613-616.

Wood ER, Dudchenko PA, Robitsek RJ, Eichenbaum H (2000) Hippocampal neurons encode information about different types of memory episodes occurring in the same location. Neuron 27:623-633.

Zilli EA, Hasselmo ME (2008) Modeling the role of working memory and episodic memory in behavioral tasks. Hippocampus 18:193-209. 